\title{
EFFECTS OF BASE SHAPE TO DRAG AT TRANSONIC AND SUPERSONIC SPEEDS BY CFD
}

\section{SERDAREVIC-KADIC, S. \& TERZIC, J.}

Abstract: This work is focused on numerical simulations of air flow around a projectile in order to determine the influence of base shape on the drag coefficient. Simulations are made for transonic and supersonic speeds of air flow. Several shapes of projectile base is considered and the results are compared to drag coefficient of projectile with flat base. Base drag component can be as high as 50\% or more of drag and any reduction in base drag can have a large payoff in increased range. Base drag is depended by flow characteristics and geometrical parameters. Influence of base shape to drag coefficient of projectile is studied by commercially CFD code FLUENT.

Key words: Drag, Base, Pressure, CFD
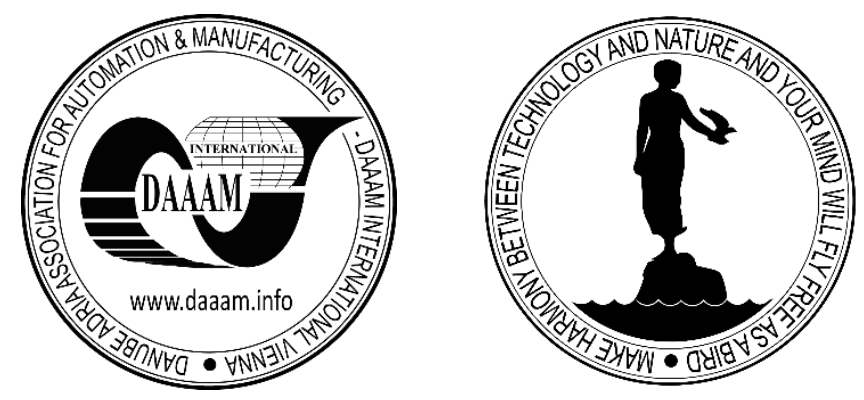

Authors' data: Assist. Prof. Dr. Sc. Serdarevic-Kadic S[abina]; Assist. Prof. Dr. Sc. Terzic, J[asmin], Faculty of Mechanical Engineering, University of Sarajevo, Vilsonovo setaliste 9, Sarajevo 71000, Bosnia and Herzegovina, serdarevic@mef.unsa.ba, terzic@mef.unsa.ba.

This Publication has to be referred as: Serdarevic-Kadic, S[abina] \& Terzic, J[asmin] (2019). Effects of Base Shape to Drag at Transonic and Supersonic Speeds by CFD, Chapter 06 in DAAAM International Scientific Book 2019, pp.071-080, B. Katalinic (Ed.), Published by DAAAM International, ISBN 978-3-902734-24-2, ISSN 1726-9687, Vienna, Austria

DOI: $10.2507 /$ daaam.scibook.2019.06 
Serdarevic-Kadic, S. \& Terzic, J.: Effects of Base Shape to Drag at Transonic and S...

\section{Introduction}

Aerodynamic problems can be solved by different methods: theoretical, experimental, (semi) empirical or their combinations. Each of these methods has its advantages and disadvantages.

Theoretical methods are based on the application of equations describing the air flow around the body. These equations are nonlinear, coupled, partial differential equations and not to have general analytical solutions (except for only a few very specialized cases). Solutions of the equations can be obtained numerically by application some of CFD codes.

According to basic sources of aerodynamic force formation, the drag of projectile consists of three components: the pressure drag or wave drag (excluding the base), the viscous drag and the base drag. Base drag, arising from flow separation at the blunt base of a body, is considerable part of projectile drag and it can be higher of $50 \%$ of total drag of projectile (Sahu, et al., 1997).

Base drag is influenced by a variety of flow and geometrical parameters. With turbulent boundary layer ahead of the base the major factors include: (1) Mach number in the free stream, just ahead of the base; (2) boundary layer momentum thickness ahead of the base; (3) base diameter; (4) angle of attack; (5) afterbody shape (boat-tail or flare angle, forebody diameter, afterbody length); and (6) parameters characterising the base drag reduction device (Suliman, et al., 2009).

At the supersonic speed, the turbulent boundary layer ahead of the base undergoes a rapid expansion at the base corner lowering the pressure in the base region; the free shear (or mixing) layer, thus formed, grows with distance from the base entraining fluid from the local free stream as well as from the base region, and is turned back to the free stream direction by the expansion wave. The static pressure in the base region is essentially constant for some distance downstream of the base. A qualitative sketch of complex dynamic regions comprising the supersonic base flow is shown at Fig. 1.

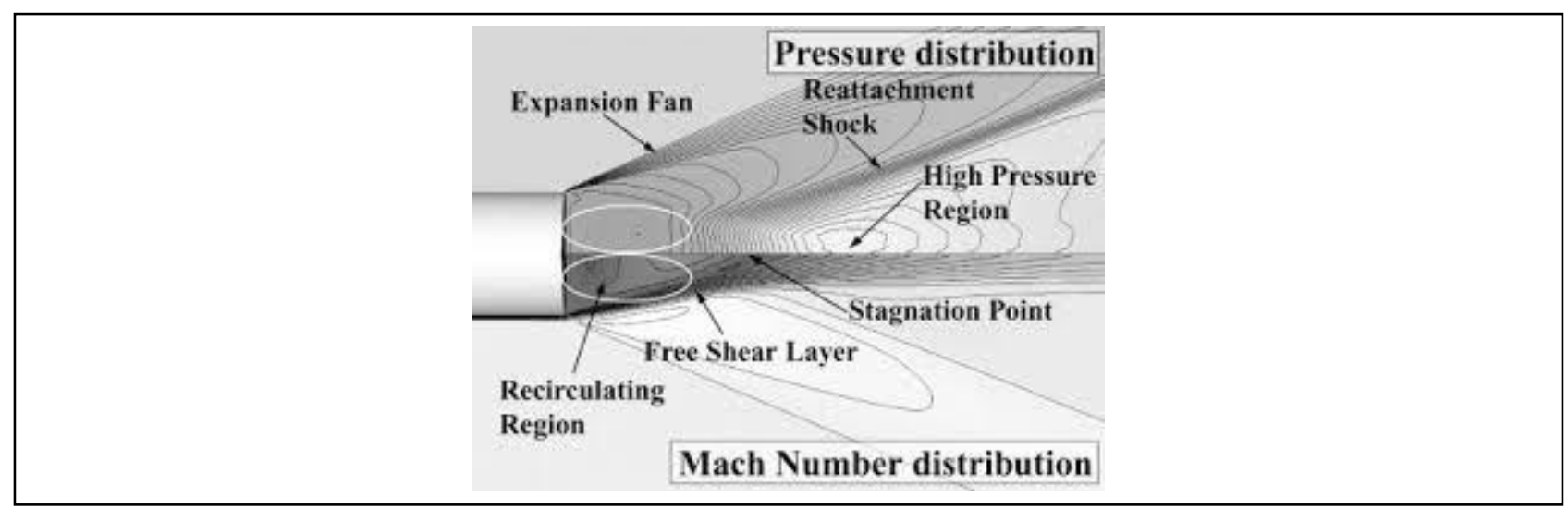

Fig. 1. Flow field behind the projectile base (Thomas, et al., 2013)

The key characteristic of this flow is the separation that occurs at the edge of base. Depending on the geometry, this separation creates either an annular or planar mixinglayer which is bounded on one side by a recirculation region. Due to discontinuity of surface, an expansion wave develops from the edge of base. 
The mixing layer converges towards a stagnation point in a high pressure region where the wake starts. The change in the direction of the streamlines in the vicinity of the stagnation point creates recompression waves. The complex dynamic interactions are inherently unsteady with high turbulent energy levels, as well as associated absolute and convective instabilities (Thomas, et al., 2013).

Measurements in separated flows are generally difficult and most experimental studies in the literature have provided only informations about the base pressure (or drag) and certain mean properties of the near-wake-like velocity profiles or static pressure variations on the wake centreline (Sivasubramanian, et al., 2006).

Some of the difficulties in the prediction of base drag have included: (1) the upstream effects of the presence of a corner in various Mach number flows at different Reynolds numbers, (2) the effects of separation, compression and/or expansion, and shock formation in the vicinity of the corner, (3) the structure of the recirculating zone, (4) the formation and structure of a mixing (shear) layer between the recirculating zone and the external flow, and (5) the effects of the configuration (e.g., boattails, fins, etc.). Detailed experimental investigations of the unsteady flow field of transonic and supersonic base flows are technically challenging and expensive.

The ability to compute the base region flow field for projectile configurations using Navier-Stokes computational techniques has been developed over the past few decades, so the Computational Fluid Dynamics (CFD) becomes an attractive alternative to expensive experiments.

Computational fluid dynamics (CFD) is the art of replacing the governing partial differenial equations of fluid flow with numbers, and advancing these numbers in space and/or time to obtain a final numerical description of the complete flow field of interest (Anderson, 1991).

Base cavity configuration is an useful choice to reduce the base drag of projectiles. The correlative experiment results show that, under the same condition, the field of fire for projectile with base cavity is larger than the projectile without base cavity $3 \sim 5 \%$ (Lu, et al. 2015).

The primary purpose of the paper is to establish the influence of base cavity shape on the drag coefficient at different speed regimes. The secondary objective is to determine how much the shape of base cavity affects to base drag coefficient for different Mach numbers.

\section{Computational Method}

Current study involves analysis of flow behind base of $155 \mathrm{~mm}$ arbitrary projectile at transonic and supersonic speeds. Numerical simulation of flow field around axisymmetric projectiles is obtained with the computational fluid dynamics software ANSYS Fluent. For all simulations is adopted following:

- The working fluid is air, an ideal gas, which is modified in accordance with compressibility and changes of thermophysical characteristics with temperature. Density and viscosity are depended of temperature and $c_{p}$ and thermal condustivity are considered as constants. 
- Pressure and temperature of air free flow correspond to parameters of air at sea level according to the standard atmosphere ICAO, $p_{\infty}=101325 \mathrm{~Pa}$ and $T_{\infty}=$ $288,15 K$.

- Flow around the projectile is compressible and turbulent.

- Discretization of spatial domain is performed by non-uniform structured mesh.

- Numerical method that simultaneously solves the equation of continuity, equation of momentum and energy equation is used. This method is developed for high velocity compressible flows.

- The governing equations are linearized by implicit manner. For a given variable, the unknown value in each cell is computed using a relation that includes both existing and unknown values from neighboring cells. Therefore each unknown will appear in more than one equation in the system, and these equations must be solved simultaneously to give the unknown quantities.

Computational domain (Fig. 2.) is limited to the projectile contour, the symmetry axis and outer boundary. Distance from outer boundary to wetted surface of projectile is more than 3 projectile length and horizontal distance from outer boundary to projectile base is 11 projectile length.

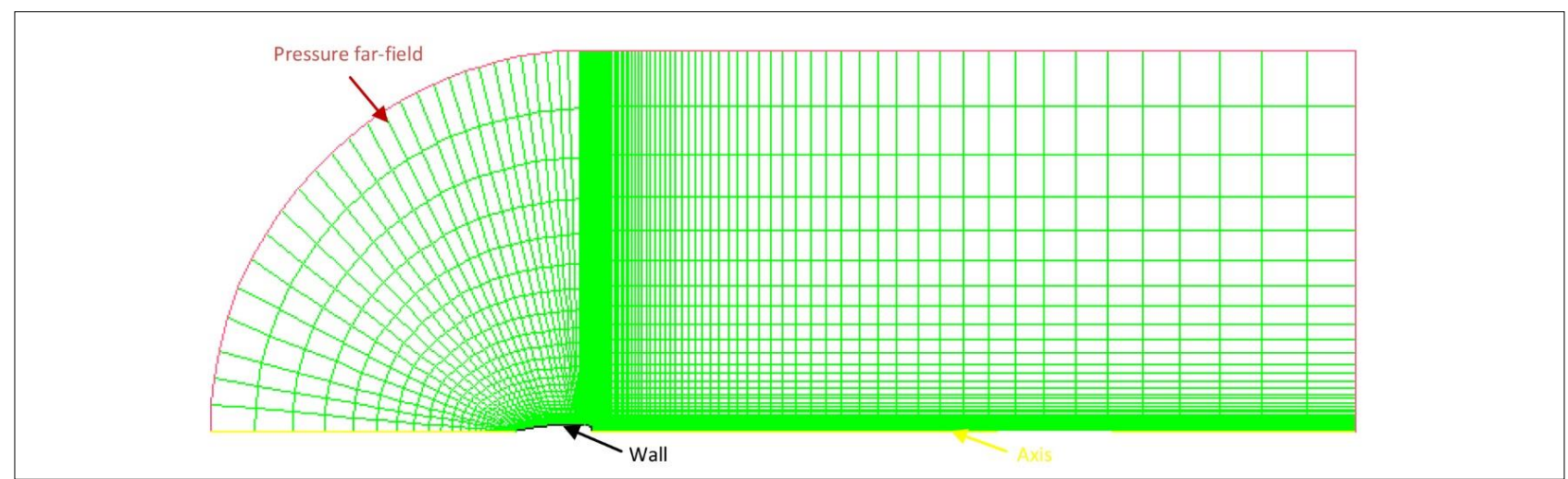

Fig. 2. Computational domain with boundaries.

Following types of boundary are chosen:

- „Wall“ boundary, which is used to delimit the regions of fluid and solid, is set to projectile contour.

- „Axis“ boundary is used as central line of axisymmeric geometry.

- „Pressure far field“ boundary, which is used for modelling of compressible free flow parameters at the infinity, is set on the outer boundary of computation domain.

Solution of Navier-Stokes equations was done by using two dimensional axisymmetric density based solver. Based on the experimental values of the drag coefficient to projectile $155 \mathrm{~mm}$ M549, turbulence model is selected. Three different turbulence models are used for simulation air flow field around projectile $155 \mathrm{~mm}$, M549: Spalart Allmaras model (SA), k- $\varepsilon$ model (k-epsilon) and Reynolds stress model (RSM). 
Numerical computations are performed for the range of Mach numbers from 0,7 to 2. Aerodynamic coefficients of drag are determined and the results for different turbulence models are shown at Fig. 3.

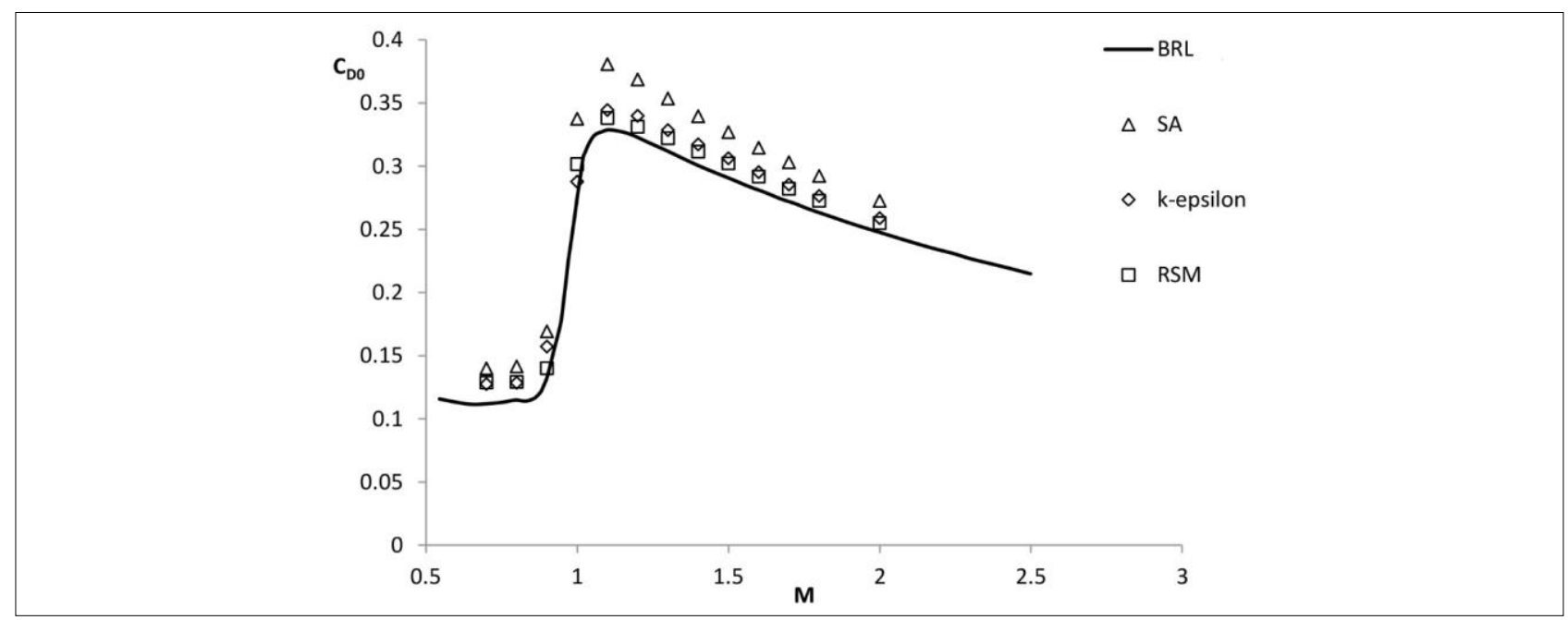

Fig. 3. Drag coefficient of projectile $155 \mathrm{~mm}$, M549, aero-range - BRL (McCoy, 1981) and estimating by CFD (SA, k-epsilon, RSM)

The results are compared with experimental data (McCoy, 1981) and Reynolds stress model of turbulence is chosen for future computations.

\section{Model geometry}

Effects of base shape on the aerodynamic coefficient of drag, at transonic and supersonic speeds for $M \geq 0,98$, are studied on the projectile $155 \mathrm{~mm}$ with aerodynamic optimized shape. Geometrical characteristics of the projectile are shown at Fig. 4.

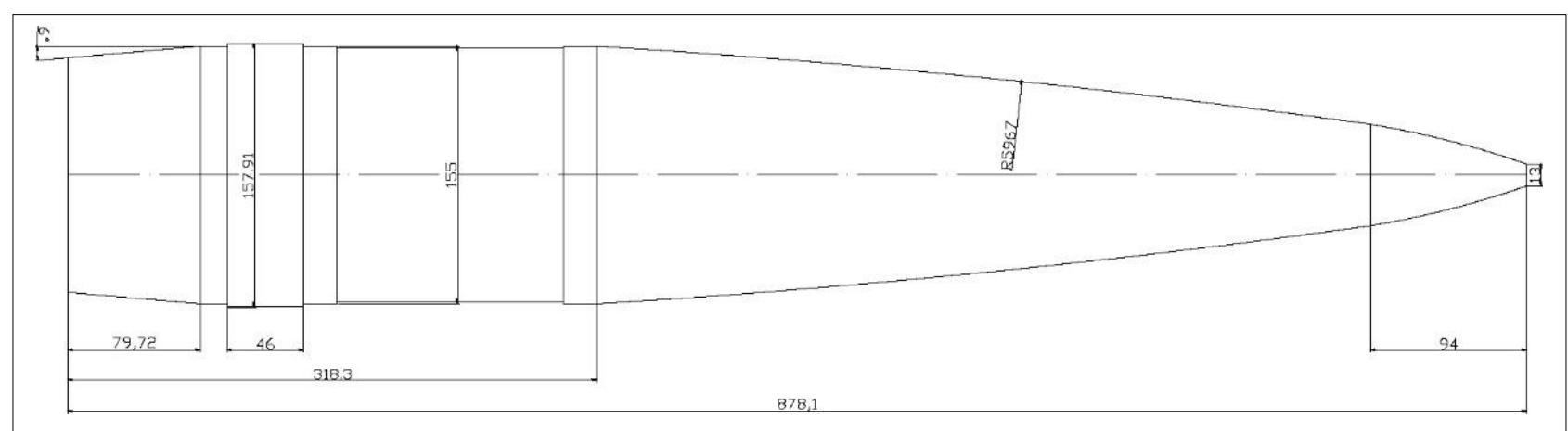

Fig. 4. Geometrical characteristics of aerodynamic optimized projectile $155 \mathrm{~mm}$

Nose of the projectile has shape of secant ogive, with finesse of ogive $\lambda_{n}=$ 3,61 . Afterbody of the projectile has shape of blunt cone with finesse ratio $\lambda_{b t}=0,51$. Total length (in calibres) of projectile is $\lambda=5,66$. Three shapes of projectile base are considered: a) flat base, b) cylindrical base cavity and c) dome base cavity (Fig. 5.). Projectile with flat base is adopted as basic configuration and reduction of drag and base drag coefficients has been studied in relation to this configuration. 
Serdarevic-Kadic, S. \& Terzic, J.: Effects of Base Shape to Drag at Transonic and S...

Base cavity in the form of sharp edges cylinder is chosen as the shape of projectile base cavity to study possible reduction of base drag. Radius of cylindrical cavity is $r_{c}=62,4 \mathrm{~mm}$ and height of cylindrical cavity is $h_{c}=10 \mathrm{~mm}$. Dimensions of dome base cavity, as another configuration, are obtained by scaling boat tail and base of projectile $155 \mathrm{~mm}$, M864.

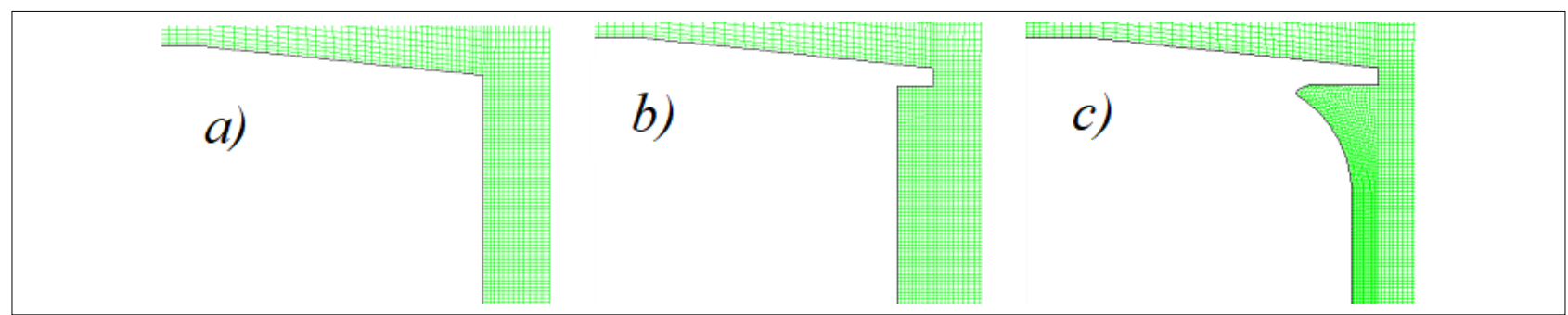

Fig. 5. Base shape: a) flat base, b) cylindrical base cavity and c) dome base cavity.

In order to construct the profile of projectile using CAD tools we must translate the architecture presented before into (x, y) points to be imported and used for further computing (Vilag, et al. 2008).

\section{Results and discussion}

A series of calculations have been made for aerodynamic optimised projectile with different base shape (flat base, cylindrical base cavity and dome base cavity). The Mach numbers computed range was $0,98 \leq M \leq 2,5$, with zero angle of attack. Flow fields around projectile basic configuration as contours of Mach number, for velocities of air free flow $M=1,08$ (transonic regime) and $M=2$ (supersonic regime) are shown at Fig. 6.

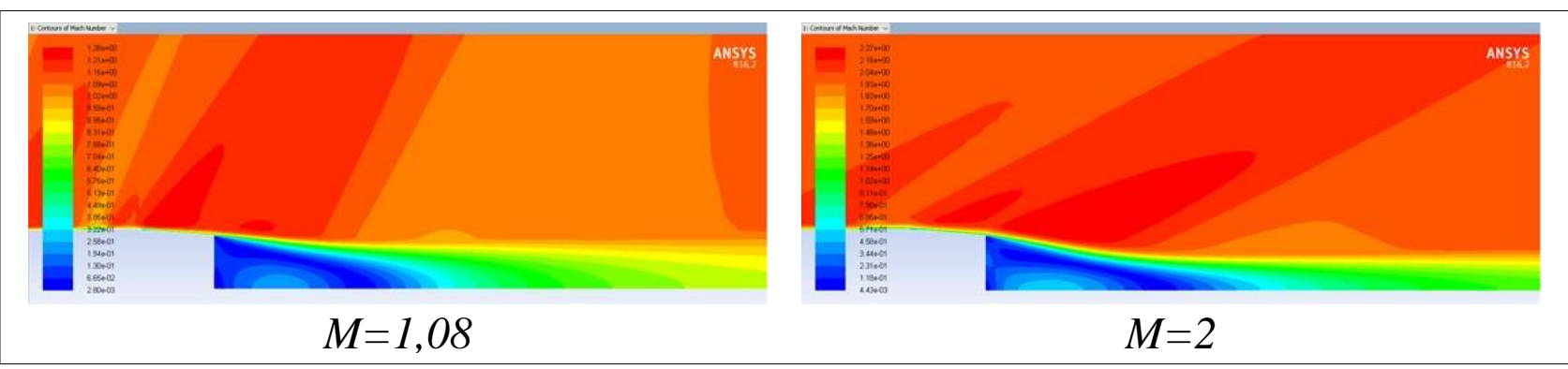

Fig. 6. Flow field around projectile with flat base at transonic and supersonic velocity

For basic configuration of projectile, computations have been performed for the following Much numbers in transonic region: 0,$98 ; 1,01 ; 1,08 ; 1,1 ; 1,15$ and 1,25 in order to determine the maximum drag coefficient. Changes of drag coefficient and base drag coefficients with Mach number for the basic configuration of projectile are shown at Fig. 7. Base drag coefficient constitutes a major portion of the drag coefficient and for the basic configuration of projectile at the transonic range $(0,98 \leq M \leq 1,25)$ base drag is about $49,4 \%$ of the overall drag experienced by the projectile. At the supersonic region $(1,25<M \leq 2,5)$, portion of base drag in the total drag is about $47,35 \%$ on average. 


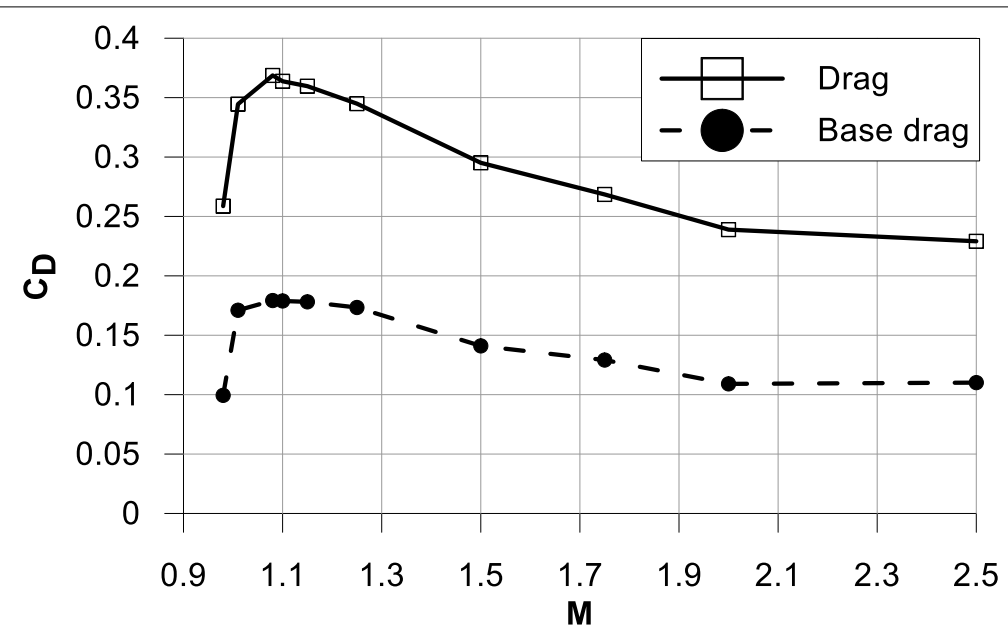

Fig. 7. Drag coefficient and base drag coefficient of projectile with flat base (basic configuration) as function of Mach number

The second series of simulations is done on the projectile configuration which is different from basic configuration by the base shape. Numerical simulations are made for the projectile configuration at transonic and supersonic speeds. Flow fields around projectile with cylindrical base cavity as contours of Mach number, for velocities of air free flow $M=1,08$ and $M=2$ are shown at Fig. 8 .

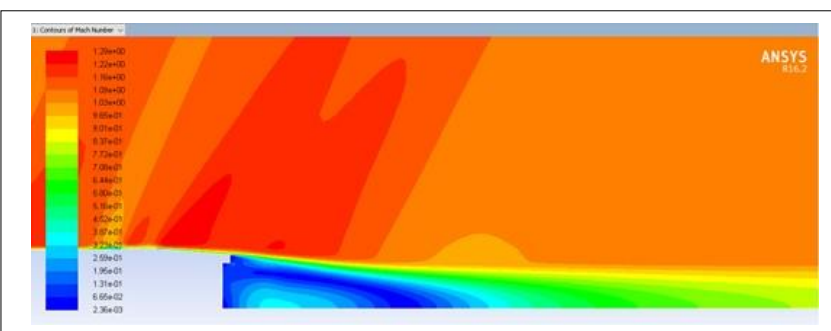

$M=1,08$

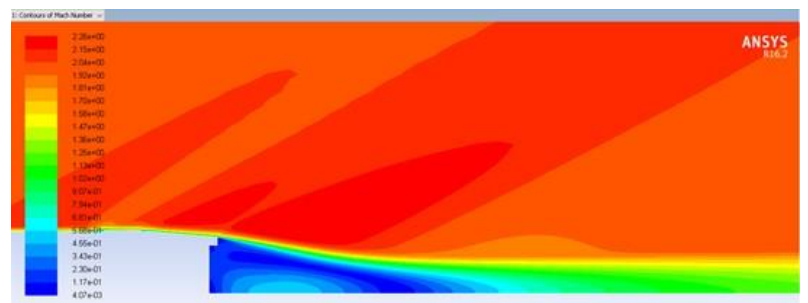

$M=2$

Fig. 8. Flow field around projectile with cylindrical base cavity at transonic and supersonic velocity

A projectile with dome base cavity is used in the third series of numerical simulations. The simulations are done for the same Mach numbers as in the previous series. Flow fields around projectile with dome base cavity as contours of Mach number, for velocities of air free flow $M=1,08$ and $M=2$ are shown at Fig. 9.

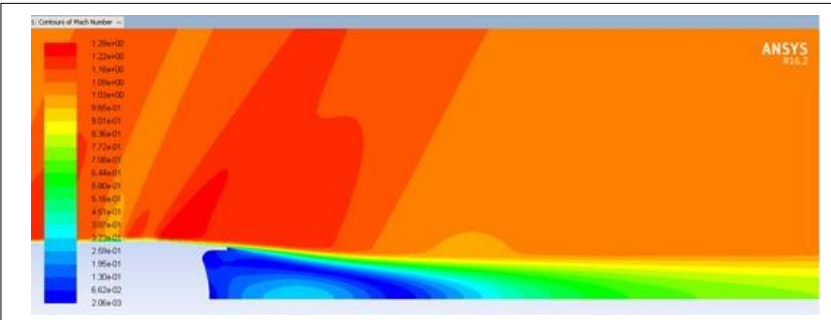

$M=1,08$

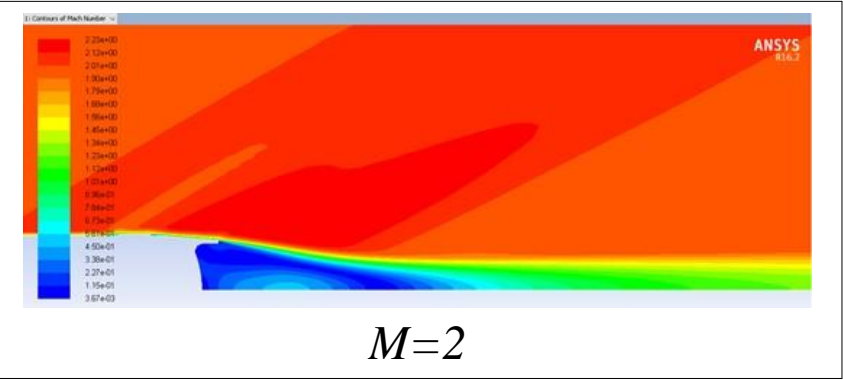

Fig. 9. Flow field around projectile with cylindrical base cavity at transonic and supersonic velocity 
Drag coefficient and base drag coefficient, for the projectile configuration with cylindrical base cavity (left) and with dome base cavity (right), as function of Mach number is shown at Fig. 10.
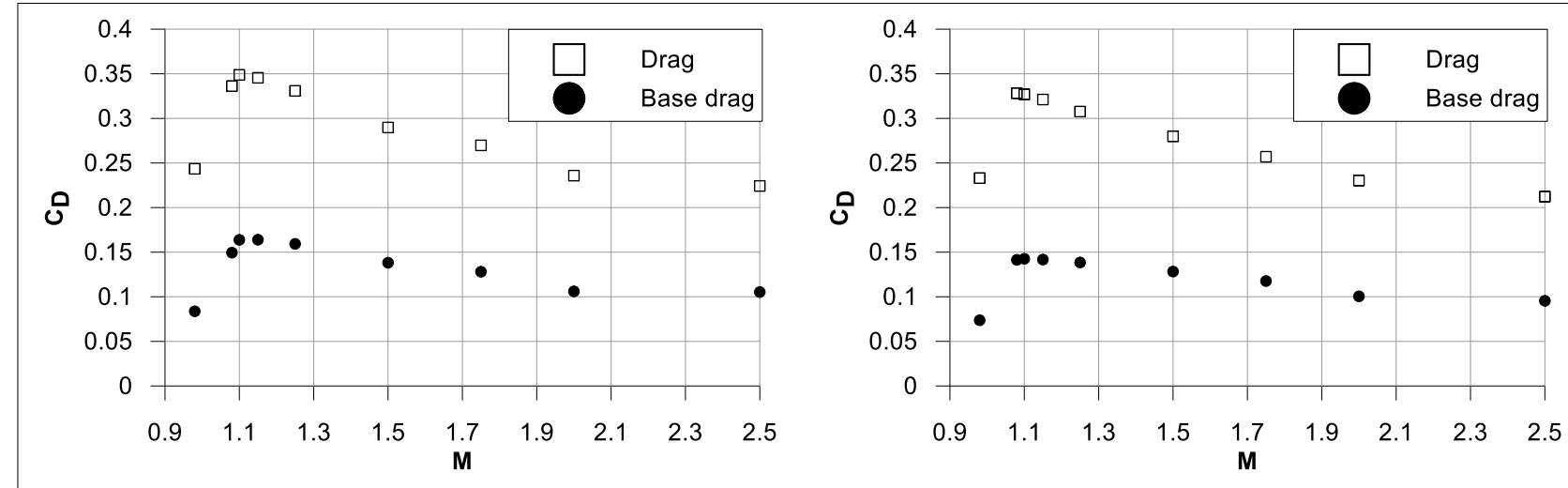

Fig. 10. Drag coefficient and base drag coefficient of projectile with cylindrical base cavity (left) and dome base cavity (right) as function of Mach number.

Radial pressure distribution on the projectile base, for cases that were studied (flat base, cylindrical base cavity and dome base cavity), at $M=1,08$ and $M=2$ are shown at Fig. 11.

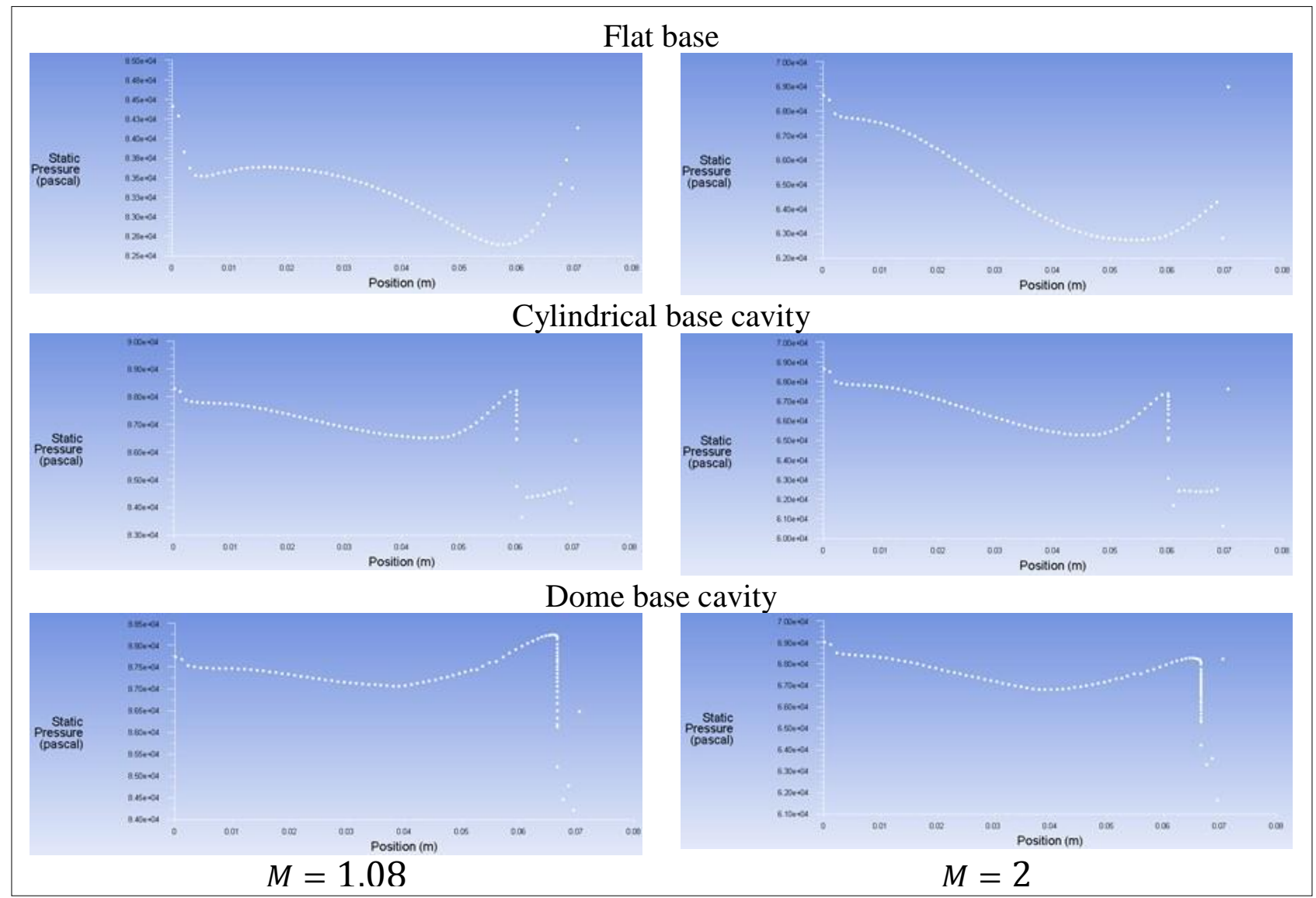

Fig. 11. Radial pressure distribution on the projectile base at $M=1,08$ and $M=2$

Radial pressure distribution on the projectile base is analysed on the base radius $r_{b}$, because at the zero angle of attack the picture of flow field is axissymmetric. 
With analysis of pressure distribution in case of projectile with a flat base, it is noticed that the curve trend of pressure for transonic speeds is different from the curve trend of pressure at supersonic speeds. The pressure is largest at the axis of symmetry at the transonic region and the pressure varies to the edge of the projectile base. It decreases to about $0,05 \cdot r_{b}$, than it lightly increases to about $0,25 \cdot r_{b}$, and than decreases again to about $0,85 \cdot r_{b}$ and again pressure increases as radius approaches the edge of base. At the supersonic region, maximum pressure at base is at the axis of symmetry too, than pressure decreases to about $0,8 \cdot r_{b}$ and than it increases. For projectile with base cavity, trend of pressure distribution on the projectile base curve is similar and for transonic and for supersonic speeds.

Reduction of projectile drag coefficient, in percentages, achieved through a change of base shape, for different Mach numbers is shown at Table 1.

\begin{tabular}{|c|c|c|c|c|}
\hline \multirow{2}{*}{$\begin{array}{c}\text { Mach } \\
\text { number }\end{array}$} & \multicolumn{2}{|c|}{$\begin{array}{c}\text { Reduction of projectile drag } \\
\text { coefficient (\%) }\end{array}$} & $\begin{array}{r}\text { Reduction of projectile base drag } \\
\text { coefficient (\%) }\end{array}$ \\
\cline { 2 - 5 } & Cylindrical base & Dome base & Cylindrical base & Dome base \\
\hline 0.98 & 5.88 & 9.91 & 15.82 & 25.90 \\
\hline 1.08 & 8.80 & 10.99 & 16.61 & 21.12 \\
\hline 1.1 & 4.13 & 10.13 & 8.42 & 20.31 \\
\hline 1.15 & 3.93 & 10.71 & 7.95 & 20.45 \\
\hline 1.25 & 4.09 & 10.80 & 8.12 & 20.16 \\
\hline 1.5 & 1.82 & 5.18 & 2.03 & 9.07 \\
\hline 2. & 1.28 & 3.60 & 2.78 & 7.87 \\
\hline 2.5 & 2.12 & 7.35 & 4.41 & 13.33 \\
\hline
\end{tabular}

Tab. 1. Drag coefficient reduction by base shape changing

The average reduction of the base drag for the projectile with cylindrical base cavity at transonic is about $11,4 \%$ and at supersonic is about $2,5 \%$ compared to basic configuration. Average reduction of drag coefficient of projectile by cylindrical base cavity at transonic regime is about 5,36\% and at supersonic regime is only $1,2 \%$.

From the results of these simulations it is noticed significant reduction of the base drag at transonic speeds and it is at range from $20,16 \%$ to $25,9 \%$. The reduction of the base drag is lower at supersonic speeds and in average it is $9,8 \%$. Significant reduction of the total drag coefficient is achieved at projectile with dome base cavity and the reduction is about 10,5\%, in average, at transonic speeds. At supersonic region, reduction of total drag coefficient is about $5,1 \%$.

\section{Conclusion}

Three series of numerical computations have been made for the projectile at transonic and supersonic speeds. In this series considered the projectiles whose external shape differ only in the shape of the projectile base. The first series of computations is related to projectile with flat base, adopting as a basic configuration. 
Drag coefficient reduction by base shape changing is analysed with respect to the basic configuration of projectile.

It was confirmed that the application of base cavity at transonic and supersonic speeds, reduces base drag coefficient, and therefore the total drag coefficient of projectile. It was noted that the reduction of aerodynamic drag coefficient of projectile higher in case of dome base cavity than in the case of cylindrical cavity base. The highest drag reduction using base cavity is realized at transonic speeds. It can also be concluded that the application of base cavity achieves uniform radial pressure distribution.

Future work will be implementation of the results in a prediction trajectory model to establish effects of base cavity shape to range for different initial conditions.

\section{References}

Anderson, J. D. (1991). Fundamentals of aerodynamics, McGraw-Hill International editions.

Lu, H. B. \& Tian. S. Y. (2015). Numerical Investigation on Flow Field of the Projectile with Base Cavity, International Conference of Electrical, Automation and Mechanical Engineering.

McCoy, R. (1981). MC DRAG - A Computer Program for Estimates the Drag Coefficients of Projectiles, Technical Report ARBRL-TR-02293, USA Ballistic Research Laboratories, Aberdeen Proving Ground, Maryland.

Sahu, J. \& Heavey, K. R. (1997). Numerical Investigation of Supersonic Base Flow with Base Bleed, Journal of Spacecraft and Rockets, Vol. 34, No. 1, pp. 62-69.

Sivasubramanian, J. \& Fasel. H. F. (2006). LES and DES of High Reynolds Number, Supersonic Base Flows with Control of the Near Wake, HPCMP Users Group Conference (HPCMP-UGC'06) 0-7695-2797-3/06 IEEE

Suliman, M. A.; Mahmoud, O. K.; Al-Sanabawy, M.A. \& Abdel-Hamid, O. E. (2009) Computational Investigation of Base Drag Reduction for a Projectile at Different Flight Regimes, In: 13th International Conference on Aerospace Sciences \& Aviation Technology, Military Technical College, Kobry Elkobbah, Cairo, Egypt

Thomas, B. Gatski \& Jean-Paul Bonnet. (2013). Compressibility, Turbulence and High Speed Flow, ISBN 978-0-12-397027-5, Academic Press, 2nd edition

Vilag, V.; Popescu, J.; Petcu, R.; Bogoi, A. \& Silivestru, V. (2008). Numerical study of an axial gas turbine, Annals of DAAAM for 2008 \& Proceedings of the 19th International DAAAM Symposium, pp.1475-1476. 